\title{
Evaluation of the role of the neonatal nurse practitioner in resuscitation of preterm infants at birth
}

\author{
W R Aubrey, C W Yoxall
}

\begin{abstract}
Background-Changes in the work patterns and numbers of medical staff in training grades pose a significant challenge to those responsible for the provision of an effective clinical neonatal service. Advanced neonatal nurse practitioners (ANNPs) may have a role in this changing neonatal service. The effectiveness of the ANNP has been established in North America but has not been properly evaluated in the United Kingdom.

Aim-To evaluate the effectiveness of ANNPs in resuscitation of preterm babies at birth against the standard set by junior medical staff.

Setting-Regional neonatal intensive care unit.

Method-Retrospective analysis of resuscitation details, other basic data, and clinical outcomes of 245 preterm $(<33$ weeks gestation) babies born in Liverpool Women's Hospital between January 1998 and April 1999.

Results-Resuscitation teams led by ANNPs provided the same resuscitation interventions as those provided by medically led teams. Although babies resuscitated by ANNP led teams were no more likely to be intubated, they were intubated more quickly and received surfactant sooner $(p=0.0001)$ than babies resuscitated by medically led teams. Babies attended by ANNP led teams were less likely to be hypothermic on admission to the neonatal unit $(p=0.013)$.

Conclusion-ANNPs are effective in the resuscitation of preterm babies at birth. (Arch Dis Child Fetal Neonatal Ed 2001;85:F96-F99)
\end{abstract}

Keywords: resuscitation; nurse practitioner; management; preterm

Traditionally, the medical workload on neonatal units in the United Kingdom has been met by small numbers of consultants supervising the work of a larger body of training grade doctors. Legislative changes have led to significant reductions in the number of hours worked by junior doctors on neonatal units in the United Kingdom over recent years. This has resulted in fewer numbers of medical hours available to cover some units and has also led to a reduction in the first hand exposure of each trainee to clinical problems. It has also been projected that paediatric specialist registrar posts in the United Kingdom will reduce in number by about $50 \%$ over the next five years. ${ }^{1}$ These changes may make it difficult for units to ensure the availability of sufficient numbers of medical staff with sufficient training and experience in clinical neonatology to meet clinical demand. The improvements in outcome for babies born prematurely seen over the last two decades have led to high expectations of neonatal services from professionals and consumers. A potential solution to this problem of increased expectation with a reduction in the availability of adequately skilled junior medical staff is the development of the role of the advanced neonatal nurse practitioner (ANNP). We have developed an ANNP service to this end in Liverpool.

Since the introduction of the ANNP, several studies from the United States have attempted to show the effectiveness of the role. ANNPs there are considered by both the medical and nursing bodies to be extremely effective and efficient in performing a challenging role. $^{2}$ However, many evaluations of the ANNP have been limited to descriptive studies using questionnaires and surveys. ${ }^{3}$ Similarly, studies of the ANNP role in this country have predominantly used survey methods to examine the distribution and function of the ANNP, and also to examine the medical, nursing, and parent view of the role. ${ }^{4-6}$

The aim of this study was to audit the skills of ANNPs in the resuscitation of preterm babies at birth, using the performance of medical staff as a standard. We also aimed to compare the clinical outcomes of infants resuscitated by ANNPs with those of infants resuscitated by medical staff.

\section{Methods}

DESCRIPTION OF THE ANNP ROLE IN PRETERM RESUSCITATION

ANNPs have been attending preterm deliveries at our hospital since 1994. The number of people contributing to the service has been increasing, but, during the period of data collection, the ANNP service was only available in the daytime during weekdays. The babies born during the daytime may therefore have been resuscitated by a team led by an ANNP or a doctor, depending on which was on duty to cover the labour ward at that time. Cases were not selected for resuscitation by a medically led or ANNP led resuscitation team. The ANNP or senior house officer on duty to cover the labour ward would be the first point of contact for the labour ward staff. If particular problems were expected, then additional support would be sought by this person from 
Table 1 Classification of resuscitation levels and proportion of babies in each group receiving each level as their maximum intervention

\begin{tabular}{lll}
\hline Resuscitation level & $\begin{array}{l}\text { Medically } \\
\text { lead teams }\end{array}$ & $\begin{array}{l}\text { ANNP } \\
\text { lead teams }\end{array}$ \\
\hline 1: Drying, stimulation, facial $\mathrm{O}_{2}$ & $26 \%$ & $21 \%$ \\
2: Mask ventilation & $11 \%$ & $11 \%$ \\
3: Intubation and ventilation & $51 \%$ & $55 \%$ \\
4: Intubation, cardiac massage, drugs & $12 \%$ & $13 \%$ \\
\hline
\end{tabular}

ANNP, Advanced neonatal nurse practitioner.

an experienced ANNP or a more senior medical colleague. Babies born at night were resuscitated by a medically led team.

DATA COLLECTION

This was a retrospective analysis of routinely collected clinical data from babies born at less than 33 completed weeks gestation between 1 January 1998 and 30 April 1999. Data collected included basic details, resuscitation details, temperature on admission to the neonatal unit, and important clinical outcomes. The maximal level of resuscitation given to each baby was defined using the classification shown in table 1 .

Data from babies for whom the resuscitation had been led by an ANNP were compared with data from those for whom a doctor had led the resuscitation. If resuscitation problems were expected, a junior ANNP would request the additional attendance of an experienced specialist registrar or ANNP; similarly, a senior house officer or junior specialist registrar would request the attendance of an experienced ANNP or experienced specialist registrar. In many cases, a consultant would also be in attendance to provide further "back up" if needed. The information in the case notes did not accurately record the names and job titles of all the people involved in the resuscitation team. The person leading the resuscitation would be responsible for completing the case notes, and, on this basis, each baby was assigned to either the ANNP group or the medical group.

Analyses were made using $\chi^{2}$, MannWhitney U-tests, and multivariate analysis as appropriate.

\section{Results}

A total of 256 babies were born at less than 33 weeks gestation in the study period. Data were available on 245 babies (96\%). In 76 (32\%)

Table 2 Comparison of basic and resuscitation details of the two groups

\begin{tabular}{llll}
\hline & ANNP lead teams & Medically lead teams & p Value \\
\hline Number of infants & 76 & 169 & \\
Birth weight (g) & $1242(530-2200)$ & $1242(440-2440)$ & 0.88 \\
Gestation (weeks) & $30(24-32)$ & $29(23-32)$ & 0.17 \\
Cord pH & $7.32(6.8-7.46)$ & $7.32(6.7-7.47)$ & 0.76 \\
Apgar (1 min) & $6(0-9)$ & $6(1-10)$ & 0.32 \\
Apgar (5 min) & $9(0-10)$ & $9(0-10)$ & 0.67 \\
Caesarean section & $53 / 76$ & $84 / 169$ & 0.005 \\
Time to intubation & 2 min $(20 \mathrm{sec}-10$ & $3 \min (1 \mathrm{~min}-18$ & 0.0001 \\
Time to surfactant administration & min) & min) & \\
$\quad$ (min) & $1(3-20)$ & $10(2-150)$ & 0.0005 \\
Intubation attempts & $1(1-3)$ & $1(1-4)$ & 0.91 \\
Admission temperature $<35^{\circ} \mathrm{C}$ & $2 / 61(3 \%)$ & $25 / 145(17 \%)$ & 0.013 \\
Admission documentation completed & $63 / 76(82 \%)$ & $113 / 169(67 \%)$ & 0.015 \\
\hline
\end{tabular}

Continuous variables expressed as median (range).

ANNP, Advanced neonatal nurse practitioner.

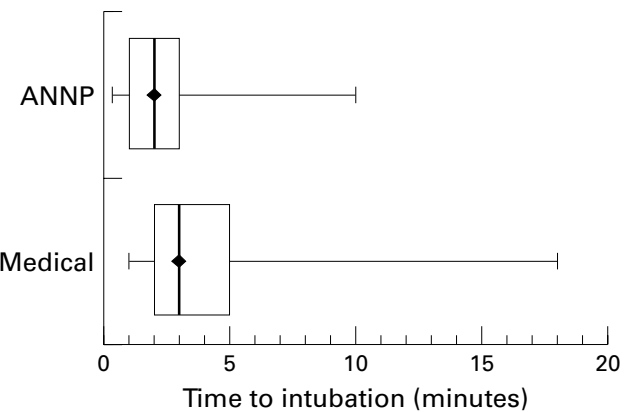

Figure 1 Time to intubation in the resuscitations led by advanced neonatal nurse practitioners (ANNPs) and medical staff. The median value is shown by the point within the box which shows interquartile range. The range is shown by the whiskers.

cases an ANNP led the resuscitation, and in $169(68 \%)$ cases a doctor led the resuscitation.

Attempts were made to find information about the whole cohort. There was no selection of infants or any attempt to match the groups. Despite this, there were no significant differences in any of the basic details, except mode of delivery (table 2). ANNPs were more likely to attend deliveries by caesarean section $(\mathrm{p}=0.005)$.

There were no significant differences in the level of resuscitation provided by the two groups (table 1). Table 2 shows further details of the resuscitation interventions. Although intubation rates were similar in the two groups of babies, the babies resuscitated by ANNP led teams were intubated more quickly than infants resuscitated by medically led teams (fig 1). Multiple linear regression analysis showed that time to intubation was independently related to being resuscitated by an ANNP led team $(p=0.003)$ and to gestational age $(p=0.029)$. Mode of delivery was not an independent predictor of time to intubation. There was no difference between the two groups in the number of intubation attempts required. Babies attended by ANNP led resuscitation teams received surfactant earlier than those attended by medically led teams.

Infants in the group attended by medically led resuscitation teams were more likely to be hypothermic (core temperature $<35^{\circ} \mathrm{C}$ ) on admission to the neonatal unit. In a multiple logistic regression analysis, hypothermia on admission was independently predicted by a medically led resuscitation team $(p=0.02)$ and birth weight $(\mathrm{p}=0.011)$.

Babies admitted to the neonatal unit after resuscitation by a medically led team were also less likely to have completed admission documentation.

There were no differences in the survival rate or the incidence of any of the other adverse clinical outcomes measured between the two groups (fig 2).

\section{Discussion}

The ANNP role is still a relatively new concept within the United Kingdom. The first British educational training course (ENB A19) was set up as recently as 1992 in Southampton. In the United States, the role has been developing since the early 1970s and is now well 


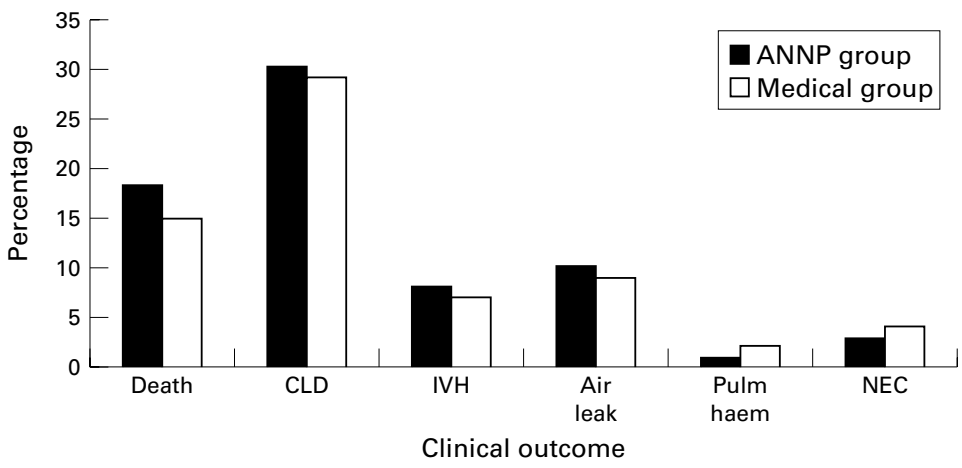

Figure 2 Long term outcome of babies resuscitated by advanced neonatal nurse practitioners (ANNPs) and doctors. CLD, Oxygen dependency beyond 28 days of life; IVH, intraventricular haemorrhage with parenchymal involvement or resulting in post haemorrhagic hydrocephalus requiring treatment; Pulm haem, pulmonary haemorrhage; NEC, necrotising enterocolitis diagnosed at laparotomy or postmortem examination.

established. The ANNP role is to assume total management of the neonate - that is, to make medical diagnoses, to order medication, and to plan management under the supervision of a neonatologist. ${ }^{7}$ The ANNP is able to instigate investigations and perform procedures previously undertaken by a doctor.

In this study we have attempted to evaluate the effectiveness of ANNPs in resuscitation of preterm babies. We believe that this is the first study in the United Kingdom to objectively quantify the effectiveness of ANNPs in this setting.

The babies attended by ANNPs in this study were more likely to have been born by caesarean section. This reflects the working patterns of the two groups. During the study period, there were insufficient ANNP numbers to provide 24 hour cover and they were responsible for neonatal resuscitation during the daytime only. It should be remembered, however, that preterm babies born by caesarean section at night are likely to be different from those born by caesarean section by day. Caesarean section for profound growth retardation, pregnancy induced hypertension, or other maternal medical problems will tend to occur in the day, whereas caesarean sections at night will usually be because of some acute emergency such as antepartum haemorrhage. As this was a retrospective, non-randomised study, the possible effects of these differences on our findings cannot be excluded. Multivariate analysis, however, does not suggest that this difference in mode of delivery between the two groups has had a major influence on the outcomes of the study.

We found that, although infants were no more likely to be intubated by ANNP led teams, those that were intubated were reported to be intubated more quickly. Although there were some outliers in the medical group, the effect of these on the overall analysis is small. The upper quartile for time to intubation in the ANNP led resuscitations (three minutes) was the same as the median time to intubation in the medically led resuscitations. The time to intubation was recorded by the person leading the resuscitation. It is possible that the difference between the two groups is a reflection of the accuracy of time keeping rather than a true difference. We do not believe that the difference in time to intubation is of any clinical significance and do not suggest that the longer time to intubation in the medically led resuscitations was evidence of poor practice. We suggest that it indicates that the ANNPs have developed a great degree of proficiency in the skill of intubation and recognition of the need for intubation, which some of the medical staff in training are still in the process of acquiring.

There was no difference between the groups in the number of attempts required to successfully intubate. However, when we looked at the whole group, we found that, for $36 \%$ of infants under $1 \mathrm{~kg}$, more than one attempt was required for successful intubation, compared with only $14 \%$ of infants over $1 \mathrm{~kg}$. When more than one attempt was needed to intubate the baby, we noted that a junior doctor or a student ANNP had attended the resuscitation with a senior colleague and had failed in one or more attempts. We suggest that, when birth weight is less than $1 \mathrm{~kg}$, the infant should be intubated and stabilised by the most experienced resuscitator in order to minimise the trauma of repeated attempts at intubation and to maximise survival potential for these fragile infants.

ANNP led teams also gave surfactant more quickly than medically led teams. The policy on our unit is to administer surfactant to all preterm babies who require intubation in the first few minutes of life, as soon as the baby is stable. This is based on studies showing beneficial effects associated with prophylactic use of surfactant. ${ }^{8}$ Although the ANNP led teams gave surfactant earlier than the medically led teams in this study, the difference was small (median time to administration eight minutes versus 10 minutes). This difference is unlikely to be clinically important as the early surfactant administration policy was adhered to in most babies.

Although the number of infants receiving external cardiac massage and/or drugs was small, the proportion was higher than we had expected. Thirty (12\%) babies received external cardiac massage, and 18 ( $7 \%$ of total) also received adrenaline. Finer et $a l^{9}$ of the Vermont-Oxford Neonatal Network have recently reported resuscitation details of 27210 babies with birth weights of $400-1500 \mathrm{~g} ; 6 \%$ of the babies in their series received cardiac compression and/or adrenaline at birth.

Previous studies discussing the use of cardiac compression and/or adrenaline in the resuscitation of preterm infants at birth have suggested that the outcome of these infants is poor. Sims et $a l^{10}$ reported that five infants of less than 28 weeks gestation requiring such treatment at birth died or were severely handicapped. Between 1989 and 1993, Rennie ${ }^{11}$ found only two normal survivors from 11 infants requiring cardiopulmonary resuscitation at birth. However, data from the Vermont-Oxford Neonatal Network show 63\% survival in 1618 infants with birth weights between $400 \mathrm{~g}$ and $1500 \mathrm{~g}$ who received cardiac compression and/or adrenaline at birth. ${ }^{9}$ 
In this study we have found that, although $15 \%$ of infants between $500 \mathrm{~g}$ and $1500 \mathrm{~g}$ received cardiac compression and/or adrenaline at birth, $73 \%$ of these infants survived, and only one survivor had a significant intraventricular haemorrhage. Survival for all infants less than 33 weeks in our study who received compression and/or adrenaline at birth was $84 \%$.

The differences in survival rates observed after these interventions is probably a reflection of the different thresholds for their use in the different studies. It is not possible to assess the effectiveness of these interventions in resuscitation or the correct threshold for their administration from retrospective studies. The guidelines for neonatal resuscitation including the use of external cardiac massage and adrenaline in our unit are broadly similar to those recommended by the European Resuscitation Council. ${ }^{12}$

We defined significant hypothermia as a core temperature on admission of less than $35^{\circ} \mathrm{C}$, as this has been shown to be an independent predictor of mortality in preterm babies. ${ }^{13}$ Babies who were attended by medically led resuscitation teams were significantly more likely to be hypothermic on admission to the neonatal unit than babies attended by ANNP led teams

We found no difference between the groups in the incidence of any major adverse long term outcome. This is not an unexpected finding. Although effective management during resuscitation at birth is vital, the impact of minor differences in practice is probably small when considered among the number of other variables that determine long term outcome in these children.

The skills required for resuscitation of the preterm newborn are not the exclusive domain of doctors. Suitably trained ANNPs can also acquire these skills. It is likely that the differences seen between the two groups in this study (shorter time to intubation and lower incidence of hypothermia on admission in the
ANNP group) reflect the longer experience of neonatal care of the ANNPs than some of the doctors in our service. It was not possible to analyse these short term outcomes by level of experience or length of service because we did not have information about the rest of the make up of the resuscitation team in each instance.

This study shows that, by introducing the ANNP into the neonatal unit, we have not compromised the quality of care provided during resuscitation at birth. ANNPs are proficient in resuscitation of preterm infants and the outcome of infants resuscitated by ANNPs is comparable to the outcome of infants resuscitated by doctors.

1 Royal College of Paediatrics and Child Health. The medical workforce in paediatrics and child health 1997-1998. London: Royal College of Paediatrics and Child Health, 1998.

2 Schultz JM, Liptak GS, Fioravanti J. Nurse practitioner 80 .

3 Mitchell-DiCenso A, Guyatt G, Marrin M. A controlled trial of nurse practitioners in neonatal intensive care. Pediatrics 1996;98:1143-8.

4 Dillon A, George S. Advanced neonatal nurse practitioners in the United Kingdom: where are they and what do they do? F Adv Nurs 1997;25:257-64.

5 Redshaw M, Harris A. Breaking new ground: an exploratory study of the role and education of the advanced neonatal nurse practitioner. London: English National Board for Nursing Midwifery and Health Visiting, 1995;15.

6 Redshaw M, Harris A. Evaluating the outcomes of advanced neonatal nurse practitioner programmes. London: English National Board for Nursing Midwifery and Health Visiting, 1996;36.

7 Mitchell A, Watts J, Whyte R. Evaluation of graduating neonatal nurse practitioners. Pediatrics 1991;88:789-94.

8 Soll RF, Morley CJ. Prophylactic surfactant vs treatment with surfactant. Cochrane Database 1997.

9 Finer NN, Horbar JD, Carpenter JH. Cardiopulmonary resuscitation in the very low birth weight infant: the Vermont Oxford Network Experience. Pediatrics 1999;104:428-34.

10 Sims DG, Heal CA, Bartle SM. Use of adrenaline and atropine in neonatal resuscitation. Arch Dis Child Fetal Neonatal Ed 1994;70:F3-9.

11 Rennie JM. Perinatal management at the lower margin of viability. Arch Dis Child Fetal Neonatal Ed 1996;74:F21418

12 Kattwinkel J, Niermeyer S, Nadkarni V, et al. An Advisory Statement from the Pediatric Working Group of the International Liason Committee on Resuscitation. Pediatrics 1999;103:e56.

13 Richardson DK, Gray JE, McCormick MC, et al. Score for Neonatal Acute Physiology: a physiologic severity index for neonatal intensive care. Pediatrics 1993;91:617-23. 